\title{
La gestione clinica del paziente con nefrolitiasi: sono necessari trials ad hoc
}

Commento all'articolo: Medical management to prevent recurrent nephrolithiasis in adults: a systematic review for an American College of Physicians Clinical Guideline

\author{
Domenico Rendina, Gianpaolo De Filippo, Pasquale Strazzullo \\ Dipartimento di Medicina Clinica e Chirurgia, Università degli Studi di Napoli “Federico II”, Napoli
}

\begin{abstract}
Nell'aprile 2013, sulla prestigiosa rivista Annals of Internal Medicine, Fink et al hanno presentato il risultato della prima metanalisi finalizzata alla definizione della corretta strategia terapeutica da assumere in caso di nefrolitiasi per impedirne la recidiva (1). Gli Autori esprimono molto chiaramente le conclusioni cliniche della loro ricerca ma altrettanto correttamente riportano gli evidenti limiti della produzione scientifica prodotta sull'argomento, dedicandovi una buona parte della discussione. Se da un lato la comunità scientifica internazionale ha prodotto dal 1977 ad oggi oltre 5000 articoli originali orientati alla definizione degli aspetti fisiopatologici della nefrolitiasi e al suo inquadramento clinico, dall'altro nello stesso intervallo temporale sono stati prodotti solo 28 studi prospettici riguardanti l'ottimizzazione della sua gestione terapeutica e di questi solo 4 articoli sono stati considerati di qualità sufficiente o buona. Questo dato stride con le stime epidemiologiche che prevedono per i prossimi anni un progressivo incremento di prevalenza, incidenza e tasso di ricorrenza della nefrolitiasi in ogni fascia di età ed etnia (2) e con le giuste attese dei pazienti affetti da nefrolitiasi che chiedono non solo perché hanno prodotto un calcolo renale ma anche cosa possono fare per evitare una recidiva o, almeno, ridurne il rischio. L'articolo di Fink et al è inoltre focalizzato sulle strategie di prevenzione della nefrolitiasi in età adulta e non bisogna dimenticare che i pediatri devono sempre più confrontarsi con questa problematica, che non riguarda più bambini ed adolescenti con anomalie congenite del metabolismo (cistinosi) o infezione delle vie urinarie (calcoli di struvite), ma sempre più bambini ed adolescenti obesi (3). È ragionevole pensare che sia proprio la multifattorialità della nefrolitiasi a rendere particolarmente difficile l'organizzazione di protocolli di ricerca e studi prospettici. Una strategia terapeutica efficace richiede la necessità di intervenire sulle abitudini di vita dei soggetti seguiti, modificando l'essenza del loro comportamento quotidiano (alimentazione, attività fisica). La sfida a cui siamo chiamati deve essere quella di trasformare queste difficoltà oggettive in un'opportunità. I dati di letteratura ci dicono che la nefrolitiasi non è una patologia d'organo o d'apparato, ma una patologia sistemica che presenta chiari collegamenti patogenetici con patologie ad elevata prevalenza, morbilità e mortalità come quelle cardiovascolari e metaboliche $(4,5)$. Proporre studi prospettici di intervento finalizzati alla prevenzione della nefrolitiasi e delle patologie cardiovascolari e metaboliche può essere uno strumento valido per motivare i pazienti alla partecipazione e per convogliare potenziali investimenti. Questo chiaramente prevede un inquadramento olistico del paziente con nefrolitiasi che richiede il coinvolgimento di figure professionali finora classicamente considerate lontane da questo ambito, quali gli esperti in scienze della nutrizione, cardiologi, endocrinologi e diabetologi.
\end{abstract}

Dichiarazione di conflitto di interesse: Gli Autori dichiarano di non avere conflitto di interessi.

Contributi economici agli Autori: Gli Autori dichiarano di non avere ricevuto sponsorizzazioni economiche per la preparazione dell'articolo.

Indirizzo degli Autori:

Dr. Domenico Rendina

Dipartimento di Medicina Clinica e Chirurgia

Università degli Studi di Napoli "Federico II"

Via Sergio Pansini 5

80131 Napoli

domenico.rendina@unina.it 


\section{Bibliografia}

1. Fink HA, Wilt TJ, Eidman KE, et al. Medical management to prevent recurrent nephrolithiasis in adults: a systematic review for an American College of Physicians Clinical Guideline. Ann Intern Med 2013;158: 535-43.

2. Dwyer ME, Krambeck AE, Bergstralh EJ, et al. Temporal trends in incidence of kidney stones among children: a 25-year population based study. J Urol 2012; 188: 247-52.

3. Matlaga BR, Schaeffer AJ, Novak TE, et al. Epidemiologic insights into pediatric kidney stone disease. Urol Res 2010; 38 : 453-7.

4. Sakhaee K. Nephrolithiasis as a systemic disorder. Curr Opin Nephrol Hypertens 2008; 17: 304-9.

5. Rendina D, De Filippo G, D'Elia L, et al. Metabolic syndrome and nephrolithiasis: a systematic review and meta-analysis of the scientific evidence. J Nephrol 2014; 27: 371-6. 